C-A/AP/\#418 Jan. 2011

\title{
Source of Second Order Chromaticity in RHIC
}

\author{
Y. Luo, X. Gu, W. Fischer, and D. Trbojevic
}

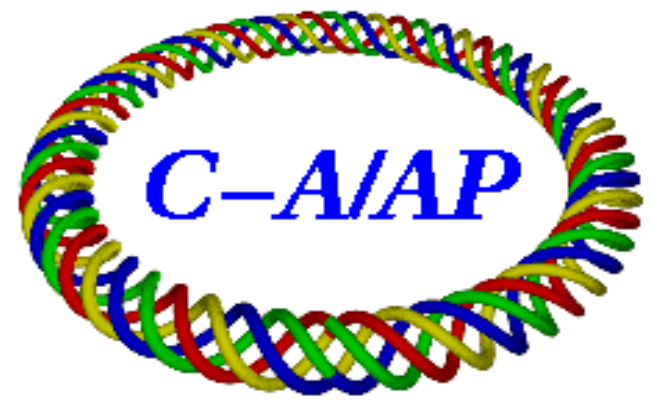

\section{Collider-Accelerator Department Brookhaven National Laboratory Upton, NY 11973}

Notice: This document has been authorized by employees of Brookhaven Science Associates, LLC under Contract No. DE-AC02-98CH10886 with the U.S. Department of Energy. The United States Government retains a non-exclusive, paid-up, irrevocable, world-wide license to publish or reproduce the published form of this document, or allow others to do so, for United States Government purposes. 


\title{
Source of second order chromaticity in RHIC
}

\author{
Y. Luo, X. Gu, W. Fischer, and D. Trbojevic \\ Brookhaven National Laboratory, Upton, NY 11973, USA
}

In this note we will answer the following questions: 1) what is the source of second order chromaticities in RHIC? 2) what is the dependence of second order chromaticity on the on-momentum $\beta$-beat? 3) what is the dependence of second order chromaticity on $\beta^{*}$ at IP6 and IP8? To answer these questions, we use the perturbation theory to numerically calculate the contributions of each quadrupole and sextupole to the first, second, and third order chromaticities.

\section{Perturbation Theory}

Based on the perturbation theory [1], the horizontal and vertical betatron tune changes due to a small quadrupole error $\Delta k_{1}(s)$ are

$$
\Delta Q_{x, y}= \pm \frac{1}{4 \pi} \oint \beta_{x, y}(s) \Delta k_{1}(s) d s .
$$

where $\beta_{x, y}(s)$ are the unperturbed horizontal and vertical amplitude functions.

The off-momentum tune shifts are

$$
\Delta Q_{x, y}=\xi_{x, y}^{(1)} \delta+\xi_{x, y}^{(2)} \delta^{2}+\xi_{x, y}^{(3)} \delta^{3} \cdots
$$

where $\delta=\Delta p / p_{0}$. The first define the first, second, and third order chromaticities are

$$
\begin{array}{r}
\xi_{x, y}^{(1)}=\frac{\partial Q_{x, y}}{\partial \delta}, \\
\xi_{x, y}^{(2)}=\frac{1}{2} \frac{\partial^{2} Q_{x, y}}{\partial \delta^{2}}, \\
\xi_{x, y}^{(3)}=\frac{1}{6} \frac{\partial^{3} Q_{x, y}}{\partial \delta^{3}} .
\end{array}
$$

For an off-momentum particle, $\Delta k_{1}(s)$ in Eq. (1) from quadrupoles and sextupoles is given by

$$
\begin{aligned}
\Delta k_{1}(s)_{x, y} & =\left[ \pm K_{1}(s) \mp K_{2}(s) D_{x}(s)\right]\left(\frac{1}{1+\delta}-1\right) \\
& =\left[\mp K_{1}(s) \pm K_{2}(s) D_{x}(s)\right]\left(\delta-\delta^{2}+\delta^{3}-\delta^{4}+\ldots\right),
\end{aligned}
$$

where $K_{1}$ and $K_{2}$ are the nominal strengths of quadrupoles and sextupoles, $D_{x}(s)$ is the horizontal dispersion.

Plugging Eq. (6) into Eq. (1) and only keep the terms of $\delta$, we obtain the first order chromaticities

$$
\xi_{x, y}^{(1)}=\frac{1}{4 \pi} \oint \beta_{x, y}(s)\left[\mp K_{1}(s) \pm K_{2}(s) D_{x}(s)\right] d s .
$$

Take derivative of Eq. (7) with respect to $\delta$ and keep the terms of $\delta^{2}$ in Eq. (6), we obtain the second order chromaticities [2]

$$
\xi_{x, y}^{(2)}=-\frac{1}{2} \xi_{x, y}^{(1)}+\frac{1}{8 \pi} \oint\left[\mp K_{1} \pm K_{2} D_{x}\right] \frac{\partial \beta_{x, y}}{\partial \delta} d s+\frac{1}{8 \pi} \oint \pm K_{2} \beta_{x, y} D_{x}^{(2)} d s .
$$

Similarly, the third order chromaticities are

$$
\begin{aligned}
\xi_{x, y}^{(3)}= & -\frac{1}{3} \xi_{x, y}^{(2)}+\frac{1}{24 \pi} \oint\left[\mp K_{1} \pm K_{2} D_{x}\right] \frac{\partial^{2} \beta_{x, y}}{\partial \delta^{2}} d s+\frac{1}{24 \pi} \oint\left[ \pm K_{1} \mp K_{2} D_{x}\right] \frac{\partial \beta_{x, y}}{\partial \delta} d s \\
& +\frac{1}{24 \pi} \oint\left[ \pm K_{2} D_{x}^{(2)}\right] \frac{\partial \beta_{x, y}}{\partial \delta} d s+\frac{1}{24 \pi} \oint\left[ \pm K_{2} D_{x}^{(3)}\right] \beta_{x, y} d s \\
& +\frac{1}{24 \pi} \oint\left[\mp K_{2} D_{x}^{(2)}\right] \beta_{x, y} d s+\frac{1}{24 \pi} \oint \pm K_{2} D_{x}^{(2)} \frac{\partial \beta_{x, y}}{\partial \delta} d s .
\end{aligned}
$$

where we defined $D_{x}=\frac{\partial x_{c o}}{\partial \delta}, D_{x}^{(2)}=\frac{\partial^{2} x_{c o}}{\partial \delta^{2}}$, and $D_{x}^{(3)}=\frac{\partial^{3} x_{c o}}{\partial \delta^{3}}$. 


\section{Sources of chromaticities}

In this section, we will use the above Eqs. (6)-(8) to search the sources of second order chromaticities. In our studies, the 2009 RHIC $100 \mathrm{GeV}$ polarized proton (p-p) run Blue ring lattice, the 2011 RHIC $250 \mathrm{GeV}$ p-p run Blue ring lattice, and the 2010 RHIC $100 \mathrm{GeV}$ Au-Au run Yellow ring lattice are used. For the 2009 RHIC $100 \mathrm{GeV}$ p-p run Blue ring lattice and the $2010 \mathrm{RHIC} 100 \mathrm{GeV} \mathrm{Au}-\mathrm{Au}$ run Yellow ring lattice, the nominal $\beta^{*}$ at IP6 and IP8 is $0.7 \mathrm{~m}$. For the 2011 RHIC $250 \mathrm{GeV}$ p-p run Blue ring lattice, the nominal $\beta^{*}$ at IP6 and IP8 is $0.65 \mathrm{~m}$.

\subsection{Examples}

Figure 1 shows the horizontal dispersion $D_{x}$ along the ring for the 2009 RHIC $100 \mathrm{GeV}$ p-p run Blue ring lattice and the 2011 RHIC $250 \mathrm{GeV}$ p-p run Blue lattice. From Figure 1, the dispersion in the interaction region (IR) IR6 and IR 8 of the 2011 RHIC $250 \mathrm{GeV}$ p-p run Blue ring lattice is much bigger than that of the 2009 RHIC $100 \mathrm{GeV}$ p-p run Blue ring lattice.

Figure 2 shows the horizontal off-momentum $\beta$-beat $\frac{\partial \beta_{x}}{\partial \delta}$ along the ring for the 2009 RHIC $100 \mathrm{GeV}$ p-p run Blue ring lattice and the 2011 RHIC $250 \mathrm{GeV}$ p-p run Blue ring lattice. For the 2009 RHIC $100 \mathrm{GeV}$ p-p run Blue ring lattice, the amplitude of the horizontal off-momentum $\beta$-beats in IR6 and IR 8 are comparable. However, for the 2011 RHIC $250 \mathrm{GeV}$ p-p run Blue ring lattice, the horizontal off-momentum $\beta$-beat in IR8 is almost twice than that in IR6. From Eq. (7), the off-momentum $\beta$-beat plays a crucial role in the second order chromaticity contribution.

Based on Eqs. (6)-(8), we are able to calculate each quadruple or sextupole's contributions to the first, second, and third chromaticities. As an example, Figure 3 shows the contributions from each element to the first order horizontal chromaticity $\xi_{x}^{(1)}$. In this example, the 2011 RHIC $250 \mathrm{GeV}$ p-p run Blue ring lattice is used. The first order chromaticities are not corrected.

\subsection{Sources}

To localize the sources of chromaticities, the first order chromaticities are not corrected. The uncorreced horizontal and vertical chromaticities are $(-89.8,87.1),(-95.2,-94.0)$, and $(-95.6,-101.3)$ for the 2009 RHIC $100 \mathrm{GeV}$ p-p run Blue ring lattice, the 2011 RHIC $250 \mathrm{GeV}$ p-p run Blue ring lattice, and the 2010 RHIC $100 \mathrm{GeV} \mathrm{Au-Au} \mathrm{run} \mathrm{Yellow} \mathrm{ring} \mathrm{lattice.}$

Tables 1-3 list the contributions of each sections to the first, second and third order chromaticities for the above three lattices. The sections include 6 IRs and 6 arcs. We define each arc is between Q9 and Q9. Q9s is included in IRs.

Table 4 summarizes each section's contributions in percentage to the whole ring for the three above lattices. The percentage is defined as the contribution of one section divided by the absolute value of the whole ring's value. Figures 4-6 plot each section's contributions in percentage for the above three lattices.

From Table 4, IR 6 and IR 8 contribute about 47\%-58\% to the first order chromaticities, while the 4 non-colliding IRs contribute about 20\%-26\% and 6 arcs contribute about $21 \%$ - $25 \%$.

From Table 4, more than $90 \%$ of contributions to the second order chromaticities are from IRs. The contributions from 6 arcs are quite small. IR6 and IR 8 contribute about $185 \%$ and $120 \%$ to the horizonatal second order chromaticity for the 2009 and 2011 p-p run lattices, while the non-colliding IRs contribute about $-77 \%$ and $-20 \%$. For the vertical second order chromaticity, IR6 and IR 8 contribute $78 \%$ and $84 \%$ for 2009 and 2011 run lattices, while the non-colliding IRs contribute about $24 \%$ and $17 \%$. For the 2010 $\mathrm{Au}-\mathrm{Au}$ run Yellow lattice, IR6 and IR8 contribute $34 \%$ and $115 \%$ to the horizontal and vertical second order chromaticity, while the other IRs contribute $71 \%$ and $-20 \%$.

Also from Table 4, IR6 and IR 8 contribute about $71 \%$ - $86 \%$ to the third order chromaticities. The noncolliding IRs contribute about 16\% except 3\% in the vertical plane for the $2010 \mathrm{Au}-\mathrm{Au}$ run Yellow lattice. For all three lattices, less than $11 \%$ of the third order chromaticities are contributed by the arcs. 
Table 1: Section contributions to chromaticities with $2009250 \mathrm{GeV}$ p-p run Blue ring lattice ( $\xi^{(1)}$ uncorr.)

\begin{tabular}{rrrrrrr}
\hline \hline & $\xi_{x}^{(1)}$ & $\xi_{y}^{(1)}$ & $\xi_{x}^{(2)}$ & $\xi_{y}^{(2)}$ & $\xi_{x}^{(3)}$ & $\xi_{y}^{(3)}$ \\
\hline IR6 & -24.82 & -23.33 & 810.26 & 1466.45 & -531496 & -442262 \\
ARC0608 & -3.62 & -3.37 & 2.47 & -49.23 & -2849.05 & -14452.4 \\
IR8 & -24.82 & -23.33 & 811.6 & 1462.74 & -530935 & -442788 \\
ARC0810 & -3.18 & -3.77 & -25.01 & 6.07 & -36950.6 & -16592.3 \\
IR10 & -4.95 & -4.74 & -293.83 & 191.45 & -62204.2 & -57063 \\
ARC1012 & -3.62 & -3.37 & 12.03 & -37.72 & -28244.2 & -13300.2 \\
IR12 & -4.92 & -4.74 & -47.23 & 272.35 & -65457 & -53276.3 \\
ARC1202 & -3.18 & -3.77 & -41.55 & 5.64 & -39509.9 & -23153 \\
IR2 & -4.92 & -4.74 & -47.31 & 272.46 & -65546.7 & -53258.8 \\
ARC0204 & -3.62 & -3.37 & 12.03 & -37.82 & -28236.2 & -13362.7 \\
IR4 & -4.95 & -4.74 & -293.69 & 191.88 & -62151.4 & -57005.4 \\
ARC0406 & -3.18 & -3.77 & -25.06 & 6.12 & -36963.9 & -16659.7 \\
\hline SUM & -89.82 & -87.09 & 874.69 & 3750.41 & $-1.49054 \mathrm{e}+06$ & $-1.20317 \mathrm{e}+06$ \\
\hline \hline
\end{tabular}

Table 2: Section contributions to chromaticities for $2011250 \mathrm{GeV}$ p-p run Blue ring lattice ( $\xi^{(1)}$ uncorr.)

\begin{tabular}{rrrrrrr}
\hline \hline & $\xi_{x}^{(1)}$ & $\xi_{y}^{(1)}$ & $\xi_{x}^{(2)}$ & $\xi_{y}^{(2)}$ & $\xi_{x}^{(3)}$ & $\xi_{y}^{(3)}$ \\
\hline IR6 & -27.58 & -27.03 & 1041.71 & 1712 & -658670 & -560237 \\
ARC0608 & -3.57 & -3.3 & -4.11 & -54.07 & -11388.5 & -40198.8 \\
IR8 & -27.96 & -26.79 & 1629.69 & 1633.69 & -551647 & -545354 \\
ARC0810 & -3.14 & -3.72 & -8.55 & 3.01 & -36019.1 & -9845.38 \\
IR10 & -4.17 & -4.05 & -220.9 & 86.62 & -51924.5 & -34472.2 \\
ARC1012 & -3.58 & -3.31 & 30.37 & -26.74 & -35417.6 & -3097.39 \\
IR12 & -4.18 & -4.05 & -146.84 & 127.5 & -52203.1 & -42809.9 \\
ARC1202 & -3.13 & -3.72 & -22.5 & 0.83 & -38012 & -11074.9 \\
IR2 & -6.94 & -6.86 & 83.58 & 392.44 & -109158 & -116487 \\
ARC0204 & -3.58 & -3.3 & 20.27 & -25.63 & -21679.4 & -4756.59 \\
IR4 & -4.2 & -4.06 & -178.06 & 90.99 & -33438.8 & -35674.1 \\
ARC0406 & -3.14 & -3.72 & -12.79 & 5.62 & -26344.3 & -11675.7 \\
\hline SUM & -95.2 & -93.97 & 2211.86 & 3946.29 & $-1.6259 \mathrm{e}+06$ & $-1.41568 \mathrm{e}+06$ \\
\hline \hline
\end{tabular}

Table 3: Section contributions to chromaticities for 2010 100GeV Au-Au run Yellow ring lattice ( $\xi^{(1)}$ uncorr.)

\begin{tabular}{rrrrrrr}
\hline \hline & $\xi_{x}^{(1)}$ & $\xi_{y}^{(1)}$ & $\xi_{x}^{(2)}$ & $\xi_{y}^{(2)}$ & $\xi_{x}^{(3)}$ & $\xi_{y}^{(3)}$ \\
\hline IR6 & -22.87 & -25.61 & 522 & 1339.18 & -811593 & -707616 \\
ARC0604 & -4.29 & -4.01 & -58.23 & 4.26 & -47003.6 & -40115.2 \\
IR4 & -6.46 & -6.08 & 508.78 & -315.65 & -77687 & -7660.6 \\
ARC0402 & -3.76 & -4.48 & -16.44 & 35.25 & -34501.8 & -35505.5 \\
IR2 & -6.34 & -6.19 & 584.75 & 81.2 & -109208 & -23254.2 \\
ARC0212 & -4.27 & -4.02 & -33.49 & 30.17 & -38995 & -18309.4 \\
IR12 & -6.34 & -6.19 & 584.89 & 84.05 & -109397 & -21279.2 \\
ARC1210 & -3.76 & -4.48 & -16.47 & 34.84 & -34433.6 & -35053.8 \\
IR10 & -6.46 & -6.08 & 506.54 & -311 & -74223.3 & -8811.74 \\
ARC1008 & -4.29 & -4.01 & -57.96 & 4.13 & -49181.6 & -37313.3 \\
IR8 & -22.87 & -25.61 & 521.65 & 1317.19 & -811990 & -716451 \\
ARC0806 & -3.82 & -4.47 & -5.9 & -11.08 & 24394.9 & -2039.15 \\
\hline SUM & -95.58 & -101.3 & 3040.12 & 2292.57 & $-2.17382 \mathrm{e}+06$ & $-1.65341 \mathrm{e}+06$ \\
\hline \hline
\end{tabular}

\subsection{Summary}

In summary, for the above three lattices, IR6 and IR8 contributed about $50 \%$ or more to the first order chromaticities and more than $70 \%$ to the third order chromaticities. IRs contribute more than $90 \%$ to the 
Table 4: Contributions to the linear and nonlinear chromaticities

\begin{tabular}{ccccccc}
\hline \hline Sections & $\xi_{x}^{(1)}$ & $\xi_{y}^{(1)}$ & $\xi_{x}^{(2)}$ & $\xi_{y}^{(2)}$ & $\xi_{x}^{(3)}$ & \multicolumn{1}{c}{$\xi_{y}^{(3)}$} \\
\hline 2009-pp-Blue: & & & & & & \\
IR6 and IR8 & -0.55 & -0.53 & 1.85 & 0.78 & -0.71 & -0.73 \\
Other IRs & -0.21 & -0.21 & -0.77 & 0.24 & -0.17 & -0.18 \\
Arcs & -0.22 & -0.24 & -0.07 & -0.02 & -0.11 & -0.081 \\
2011-pp-Blue: & & & & & & \\
IR6 and IR8 & -0.58 & -0.57 & 1.20 & 0.84 & -0.74 & -0.78 \\
Other IRs & -0.20 & -0.20 & -0.20 & 0.17 & -0.15 & -0.16 \\
$\quad$ Arcs & -0.21 & -0.22 & 0.00 & -0.02 & -0.10 & -0.05 \\
2010-AuAu-Yellow: & & & & & & \\
IR6 and IR8 & -0.47 & -0.50 & 0.34 & 1.15 & -0.74 & -0.86 \\
Other IRs & -0.26 & -0.24 & 0.71 & -0.20 & -0.17 & -0.03 \\
Arcs & -0.25 & -0.25 & -0.06 & 0.04 & -0.08 & -0.10 \\
\hline \hline
\end{tabular}

second order chromaticities. For the p-p run lattices, IR6 and IR 8 contribute $185 \%$ and $120 \%$ of the total horizontal second order chromaticity for 2009 and 2011 run lattices. The non-colliding IRs contribute -77\% and $-20 \%$. For the $2010 \mathrm{Au}-\mathrm{Au}$ run Yellow ring lattice, each IR contribute about $20 \%$ to the horizontal second order chromaticity. IR6 and IR 8 contributes about $120 \%$ to the vertical chromaticity. For the $\xi_{x, y}^{(1)}$ uncorrected lattices, the arcs contribute about $20 \%$ to the first order chromaticities. However, their contributions to the second and third order chromaticities are small.

As a comparison, Tables 5-7 list the contributions of each sections to the first, second and third order chromaticities for the above three lattices with first order chromaticities corrected to $(1,1)$. In the correction, all focusing and defocusing sextupoles have the same strengths. Comparing Tables 1-3 and Tables 5-7, After first order chromaticity correction, the second order chromaticities do not change too much. This can be explained by the fact that the betatron phase advance of each FODO cell in the RHIC arcs are about $90^{\circ}$ [3]. The adjacent sextupoles' contribution to the second order chromaticity will cancel.

\section{Nonlinear chromaticity's dependence on on-momentum $\beta$-beat}

In this section, we will investigate the effects of on-momentum $\beta$-beat on the second order chromaticities. The on-momentum $\beta$-beat is the $\beta$ change of on-momentum particles due to quadrupole errors.

\subsection{Simulation Setup}

In the following simulation study, we will randomly assign strength errors to all the quadroples to generate on-momentum $\beta$-beat. The quadrupole errors are given in percentage. The error percentage are generated with the formula MaxErr*(rnd(1)-0.5)*2, where function $r n d(1)$ is the random number generator which produces random numbers with a uniform distribution between (0-1), MaxErr is maximum strength error in percentage. For each seed or case, we keep MaxErr same for all quadrupoles. In this study, MaxErr is chosen to be $0.0005,0.001,0.002,0.003,0.004$. We simulate 50 sets of quadrupole errors for each lattice. The first order chromaticities are corrected to $(+1,+1)$.

We will focus on the averaged on-momentum $\beta$-beat $<\Delta \beta_{x, y} / \beta_{x, y, 0}>$ along the ring. Figure 7 shows the correlation between $\left\langle\Delta \beta_{x} / \beta_{x, 0}>\right.$ and $\left\langle\Delta \beta_{y} / \beta_{y, 0}>\right.$ in this simulation studies for the above three lattices. In most cases, the horizontal and vertical on-momentum $\beta$-beats scale with each other. Therefore, in the following discussion, we will only use the averaged transverse on-momentum $\beta$-beat $\left\langle\Delta \beta / \beta_{0}\right\rangle$ which is given by $\left(<\Delta \beta_{x} / \beta_{x, 0}>+<\Delta \beta_{y} / \beta_{y, 0}>\right) / 2$. The changes of second and third order chromaticities are defined by $\Delta \xi_{x, y}^{(2)} / \xi_{x, y, 0}^{(2)}$ and $\Delta \xi_{x, y}^{(3)} / \xi_{x, y, 0}^{(3)}$. 
Table 5: Section contributions to chromatities with $2009250 \mathrm{GeV}$ p-p run Blue ring lattice ( $\xi^{(1)}$ corr.)

\begin{tabular}{rrrrrrr}
\hline \hline & $\xi_{x}^{(1)}$ & $\xi_{y}^{(1)}$ & $\xi_{x}^{(2)}$ & $\xi_{y}^{(2)}$ & $\xi_{x}^{(3)}$ & $\xi_{y}^{(3)}$ \\
\hline IR6 & -24.82 & -23.33 & 790.67 & 1572.9 & 140726 & -8334.45 \\
ARC0608 & 12.03 & 9.93 & -9.35 & 160.93 & 13412 & 86025 \\
IR8 & -24.85 & -22.14 & 1021.77 & 1415.15 & 95018.4 & 8184.07 \\
ARC0810 & 11.55 & 10.99 & -66.93 & -26.64 & 100199 & 43998.4 \\
IR10 & -4.95 & -4.74 & -295.57 & 207.47 & -26936.5 & -23463.2 \\
ARC1012 & 12.01 & 9.9 & -56.43 & 88.28 & 102326 & 57822.7 \\
IR12 & -4.85 & -3.55 & -14.57 & 197.61 & -32186.4 & -44556.3 \\
ARC1202 & 11.48 & 11.01 & 46.46 & -53.54 & 119750 & 66446.7 \\
IR2 & -4.92 & -4.74 & -59.7 & 289.56 & -24350.8 & -55498.3 \\
ARC0204 & 12.01 & 9.89 & -60.63 & 92.89 & 92814.3 & 51817.8 \\
IR4 & -4.79 & -3.56 & -254.31 & 86.03 & -27911.5 & -7192.21 \\
ARC0406 & 11.39 & 11.03 & 145.99 & -35.36 & 74747.5 & 41176.6 \\
\hline SUM & 1.3 & 0.69 & 1187.37 & 3995.3 & 627608 & 216427 \\
\hline \hline
\end{tabular}

Table 6: Section contributions to chromatities with $2011250 \mathrm{GeV}$ p-p run Blue ring lattice ( $\xi^{(1)}$ corr.)

\begin{tabular}{rrrrrrr}
\hline \hline & $\xi_{x}^{(1)}$ & $\xi_{y}^{(1)}$ & $\xi_{x}^{(2)}$ & $\xi_{y}^{(2)}$ & $\xi_{x}^{(3)}$ & $\xi_{y}^{(3)}$ \\
\hline IR6 & -27.58 & -27.03 & 966.53 & 1855.99 & 170350 & -139503 \\
ARC0608 & 12.97 & 10.91 & 39.33 & 228.84 & 43877.8 & 167318 \\
IR8 & -28 & -25.43 & 1800.88 & 1429.85 & 67221 & -127367 \\
ARC0810 & 12.47 & 12.22 & -138.97 & -4.28 & 130719 & 16042.4 \\
IR10 & -4.17 & -4.05 & -220.79 & 93.81 & -19229.4 & -12078.8 \\
ARC1012 & 13.02 & 10.99 & -130.38 & 51.16 & 152815 & 30051 \\
IR12 & -4.11 & -2.76 & -106.38 & 63.88 & -29534.9 & -27641.9 \\
ARC1202 & 12.4 & 12.26 & 38.07 & -66.57 & 151949 & 40105.5 \\
IR2 & -6.94 & -6.86 & 36.38 & 413.39 & -38680.9 & -91579.8 \\
ARC0204 & 13 & 10.94 & -99.54 & 62.35 & 91985.8 & 34165.9 \\
IR4 & -4.02 & -2.73 & -141.79 & -27.22 & -12391.4 & 8677.21 \\
ARC0406 & 12.25 & 12.24 & 159.72 & -22.74 & 47338.6 & 24241 \\
\hline SUM & 1.3 & 0.7 & 2203.04 & 4078.49 & 756419 & -77569.9 \\
\hline \hline
\end{tabular}

Table 7: Section contributions to chromatities with $2010100 \mathrm{GeV} \mathrm{Au}-\mathrm{Au}$ run Yellow ring lattice ( $\xi^{(1)}$ corr.)

\begin{tabular}{rrrrrrr}
\hline \hline & $\xi_{x}^{(1)}$ & $\xi_{y}^{(1)}$ & $\xi_{x}^{(2)}$ & $\xi_{y}^{(2)}$ & $\xi_{x}^{(3)}$ & $\xi_{y}^{(3)}$ \\
\hline IR6 & -22.87 & -25.61 & 765.5 & 1178.84 & 118473 & 80424.3 \\
ARC0604 & 12.62 & 11.21 & 176.28 & 21.82 & 171297 & 131472 \\
IR4 & -6.44 & -4.76 & 485.45 & -332.93 & -117126 & -61989.3 \\
ARC0402 & 12.13 & 12.36 & -13.76 & -59.42 & 157219 & 99023.1 \\
IR2 & -6.34 & -6.19 & 678.84 & 117.07 & -96350.8 & -51033.3 \\
ARC0212 & 12.58 & 11.23 & 156.7 & -74.35 & 191960 & 37037.7 \\
IR12 & -6.23 & -4.88 & 596.75 & 108.87 & -91019 & -25615.7 \\
ARC1210 & 11.96 & 12.38 & -60.92 & -114.7 & 152104 & 86007.5 \\
IR10 & -6.46 & -6.08 & 601.32 & -305.42 & -124078 & -52198.7 \\
ARC1008 & 12.64 & 11.22 & 195.08 & 14.11 & 184819 & 126492 \\
IR8 & -22.64 & -24.28 & 546.69 & 985.79 & 113400 & 58546.9 \\
ARC0806 & 11.97 & 12.43 & 37.1 & 16.36 & 10786.8 & 14036.1 \\
\hline SUM & 2.91 & -0.96 & 4165.07 & 1556.04 & 671485 & 442203 \\
\hline \hline
\end{tabular}

\subsection{Results}

Figure 8 plots the changes in the second order chromaticities due to the on-momentum $\beta$-beat for the above three lattices. In Figures 8, "H" represents the horizontal plane and "V" represents the vertical plane. For 
most seeds, when $\left\langle\Delta \beta / \beta_{0}\right\rangle$ is below $10 \%$, the second order chromaticity changes are less than $30 \%$. When $\left\langle\Delta \beta / \beta_{0}\right\rangle$ is below $20 \%$, the second order chromaticity changes are less then $50 \%$. For the above three lattices, the second order chromaticities without quadrupole errors are all below 4000 . Therefore, $10 \%$ onmomentum $\beta$-beat will introduce maximum of 1200 unit changes to the second order chromaticities. However, for $20 \%$ on-momentum $\beta$-beat, the second order chromaticities may change the second order chromaticities by 2000 units.

Figures 9 plots the changes in the third order chromaticities due to the on-momentum $\beta$-beat for the above three lattices. Although for the most seeds, when the averaged on-momentum $\beta$-beat is below $20 \%$, the third order chromaticity changes are less than 50\%. However, for some cases with the 2009 and 2011 p-p run Blue lattices, even if the on-momentum $\beta$-beat is about $15 \%$, the vertical third order chromaticities are already more than doubled.

\subsection{Summary}

In summary, for the above three lattices, $10 \%$ on-momentum $\beta$-beat may introduce $30 \%$ changes in second order chromaticities which is about 1200 units. According to the RHIC online second order chromaticity correction experience, to correct 1000-2000 units of second order chromaticity shoul not be very difficult. However, to correct total second order chromaticiies above 4000 units will not be straight-forward if we want to keep more or less balanced strengths among sextupole families and do not want to change the sextupole polarities. With large on-momentum $\beta$-beat, the relative phase advances between sextupoles in sextupole families will change too, which in return increases the difficulty in the online correction.

\section{Nonlinear chromaticity's dependence on $\beta^{*}$}

In this section we investigate the nonlinear chromaticity's dependence on $\beta^{*}$ at IP6 and IP8. In this study, the $2009100 \mathrm{GeV}$ p-p run Blue ring lattices and the $2010100 \mathrm{GeV}$ Au-Au run Yellow ring lattices are used. The first order chromaticities are corrected to $(1,1)$. In the process of generating these low $\beta^{*}$ lattices, we may exceed some power supplies' maximum current limits.

Figures 10 and 11 show the second and third order chromaticities as functions of $\beta^{*}$ for the $2009250 \mathrm{GeV}$ p-p run Blue ring lattices and the $2010100 \mathrm{GeV}$ Au-Au run Yellow ring lattices. From Figures 10 and 11 , the absolute values of second and third order chromaticities increase when the $\beta^{*} \mathrm{~s}$ are decreased. And in most cases, the increase in nonlinear chromaticities with $\beta^{*}$ are faster than linear growth. For the 2009 $100 \mathrm{GeV}$ p-p run Blue ring lattices, when the $\beta^{*}$ is $0.5 \mathrm{~m}$, the vertical second order chromaticity reaches 6000 and the horizontal third order chromaticity reaches $2.5 \times 10^{6}$. For the $2010100 \mathrm{GeV} \mathrm{Au}-\mathrm{Au}$ run Yellow ring lattices, when $\beta^{*}$ is $0.65 \mathrm{~m}$, the horizontal second order chromaticity reaches 5500 and the horizontal third order chromaticity reaches $1.0 \times 10^{6}$.

Figure 12 shows the second and third order chromaticities versus $\beta^{*}$ for the $2009250 \mathrm{GeV}$ p-p run Blue ring lattices with second order chromaticity correction. The second order chromaticities are corrected with the 4-knob method [4]. After second order chromaticity correction, the second order chromaticities are significantly reduced. However, the third order chromaticities have small changes.

Figure 13 and 14 shows the percentage contributions from IR6 and IR 8 to the first, second, and third chromaticities during $\beta^{*}$ squeezing for the $2009100 \mathrm{GeV}$ p-p run Blue ring lattices and the $2010100 \mathrm{GeV}$ $\mathrm{Au}-\mathrm{Au}$ run Yellow ring lattices. With smaller $\beta^{*}$, IR6 and IR 8 contribute more percentages to the first order chromaticities. However, their contribution percentages to nonlinear chromaticities do not change significantly.

\section{Acknowledgments}

We thank J. Bengtsson and C. Gardner for the stimulating discussions during this study.

\section{References}

[1] H. Wiedemann, Particle Accelerator Physics, Springer-Verlag, 1995.

[2] J. Bengtsson, The sextupole scheme for the Swiss Light Source(SLS): an analytical approach, SLS Note 9/97, March 7, 1997. 
[3] S. Tepikian, private communications.

[4] Y. Luo, et al., BNL C-AD AP Note 348, Jan., 2009. 


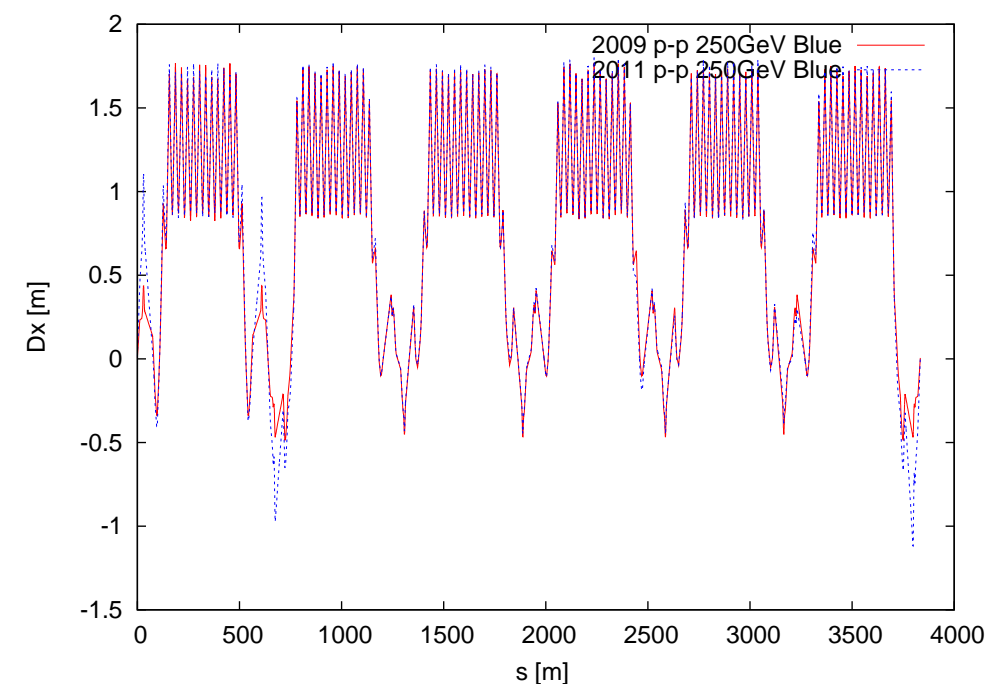

Figure 1: Dispersion along the ring for 2009 and 2011 250GeV p-p run Blue ring lattices.

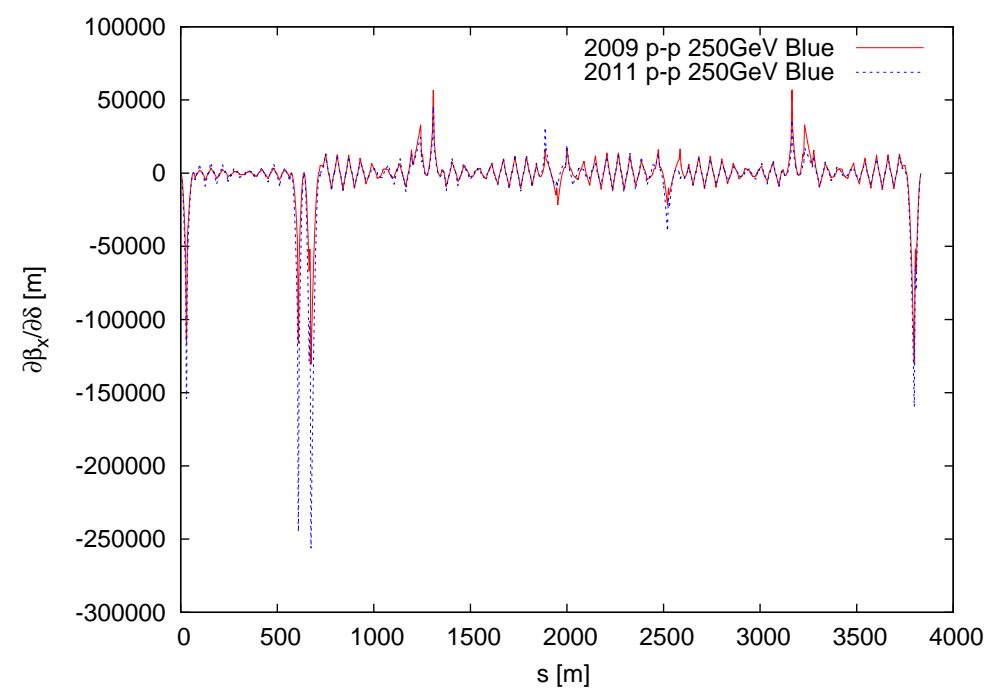

Figure 2: $\frac{\partial \beta_{x}}{\partial \delta}$ for 2009 and $2011250 \mathrm{GeV}$ p-p run Blue ring lattices.

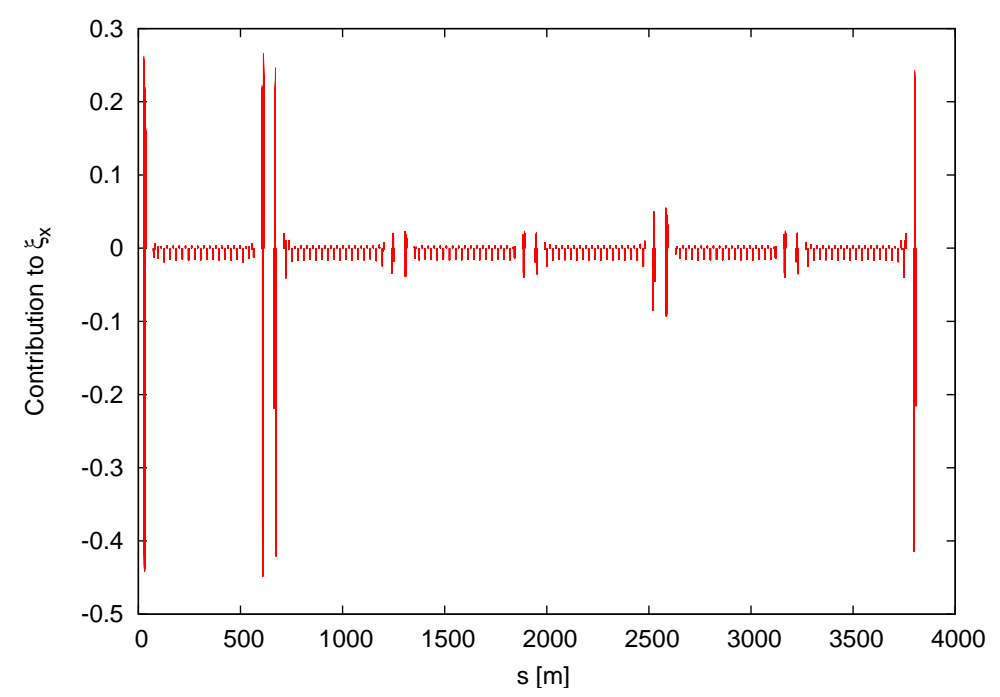

Figure 3: Each element's contribution to $\xi_{x}^{(1)}$ along the ring for $2011250 \mathrm{GeV}$ p-p run Blue ring lattice. 


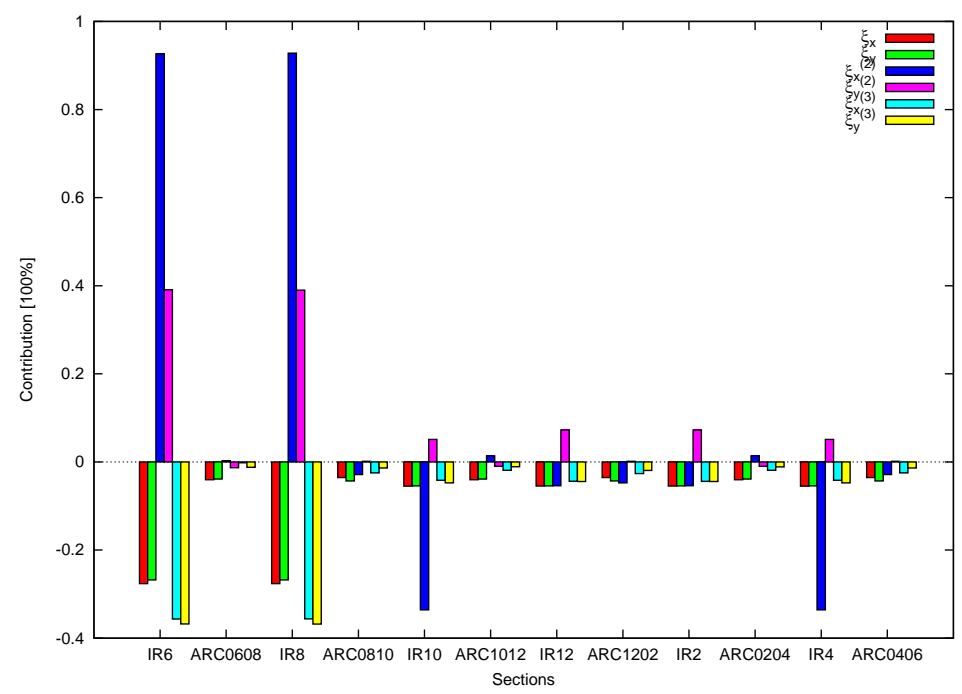

Figure 4: Section contributions to chromatities with $2009250 \mathrm{GeV}$ p-p run Blue ring lattice ( $\xi^{(1)}$ uncorr.)

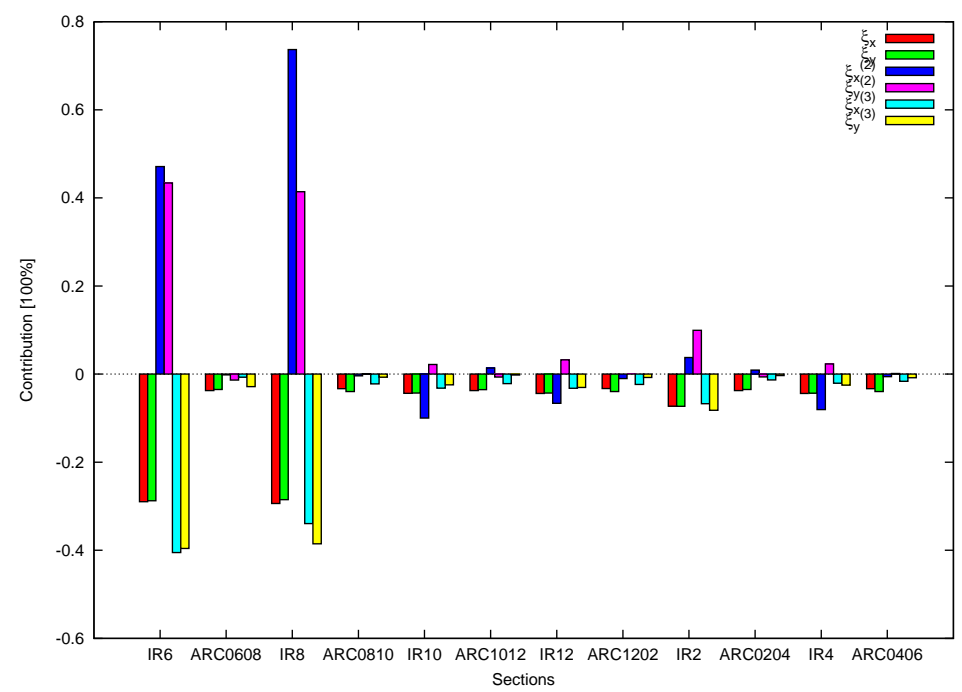

Figure 5: Section contributions to chromatities with $2011250 \mathrm{GeV}$ p-p run Blue ring lattice ( $\xi^{(1)}$ uncorr.)

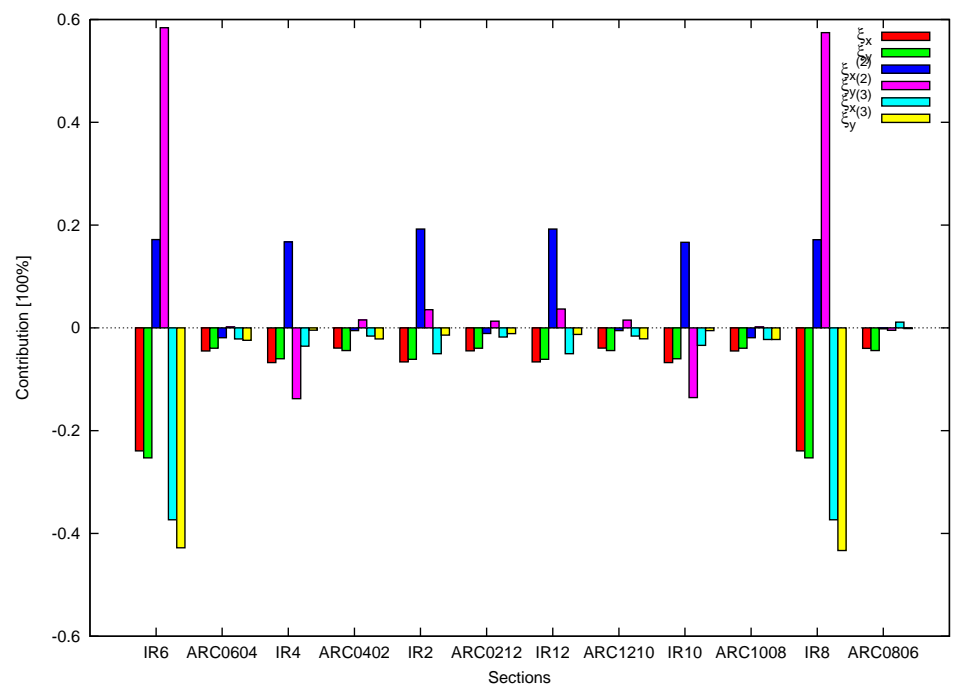

Figure 6: Section contributions to chromatities for $2010100 \mathrm{GeV}$ Au-Au run Yellow ring lattice ( $\xi^{(1)}$ uncorr.) 


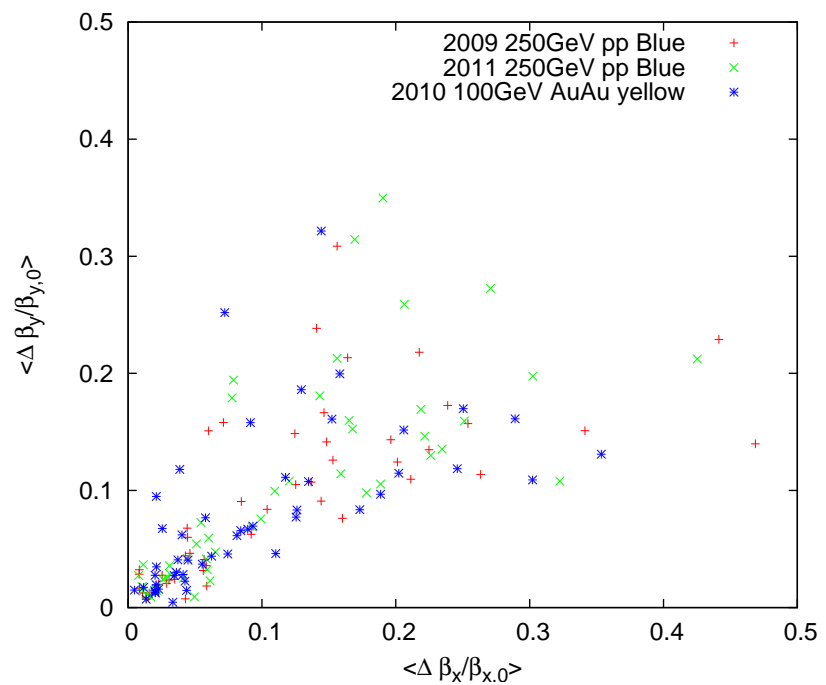

Figure 7: Correlation between $<\Delta \beta_{x} / \beta_{x, 0}>$ and $<\Delta \beta_{y} / \beta_{y, 0}>$ in this simulation studies

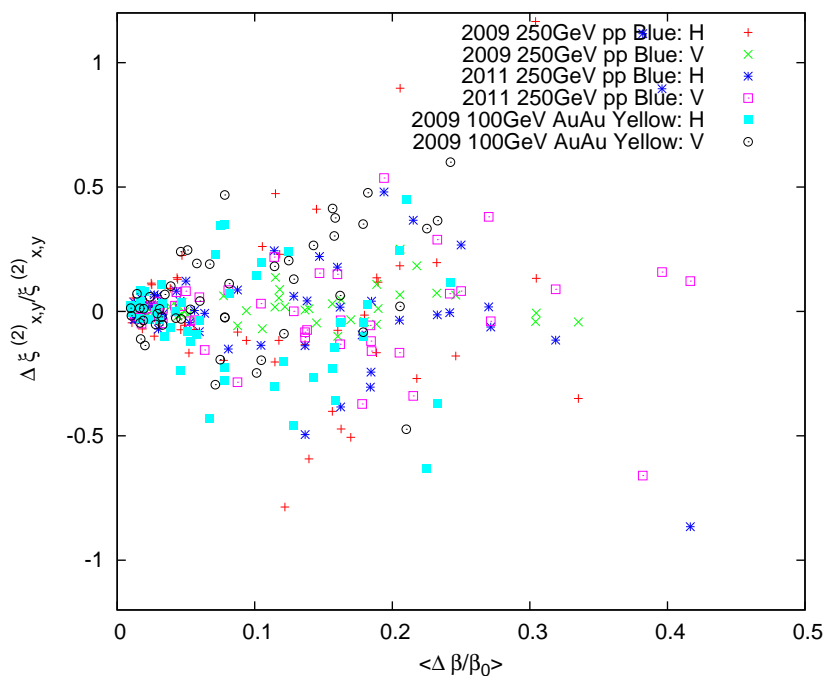

Figure 8: Second order chromaticity changes versus on-momentum $\beta$-beat

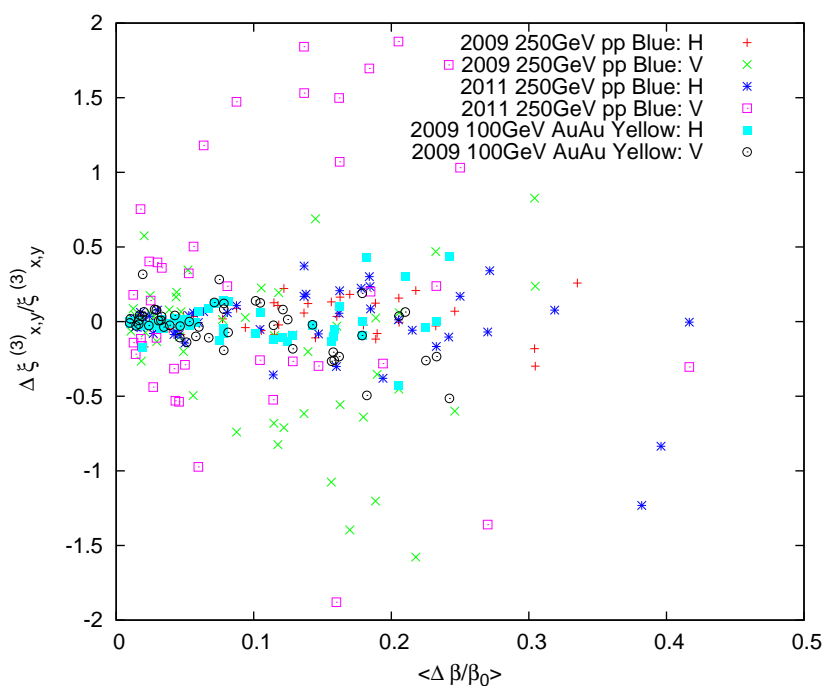

Figure 9: Third order chromaticity changes versus on-momentum $\beta$-beat 


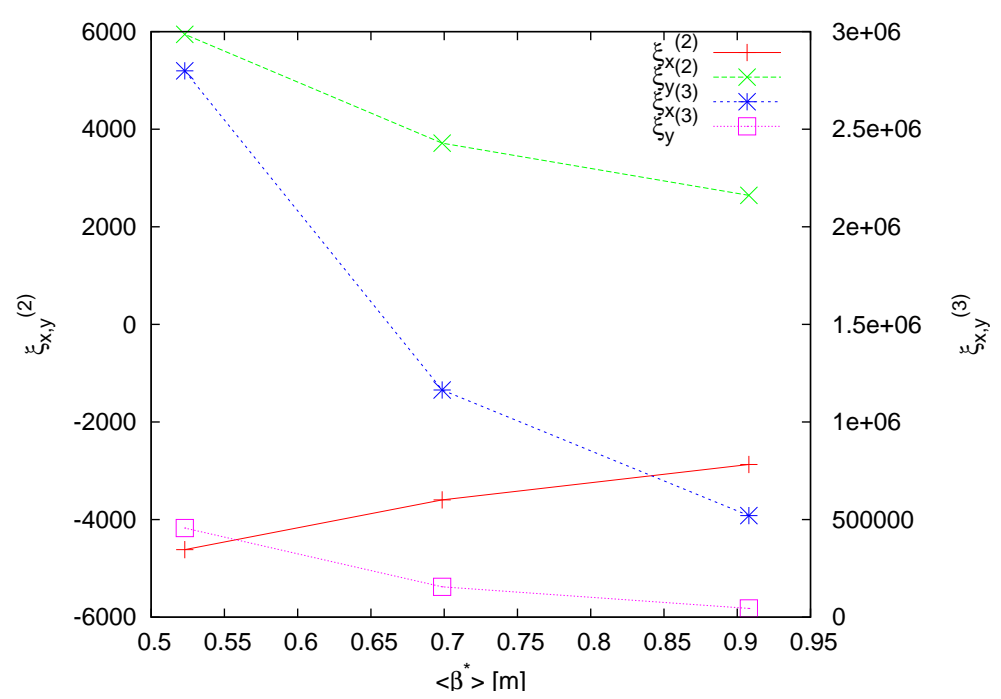

Figure 10: Chromaticities versus $\beta^{*}$ for $2009100 \mathrm{GeV}$ p-p run Blue ring lattices

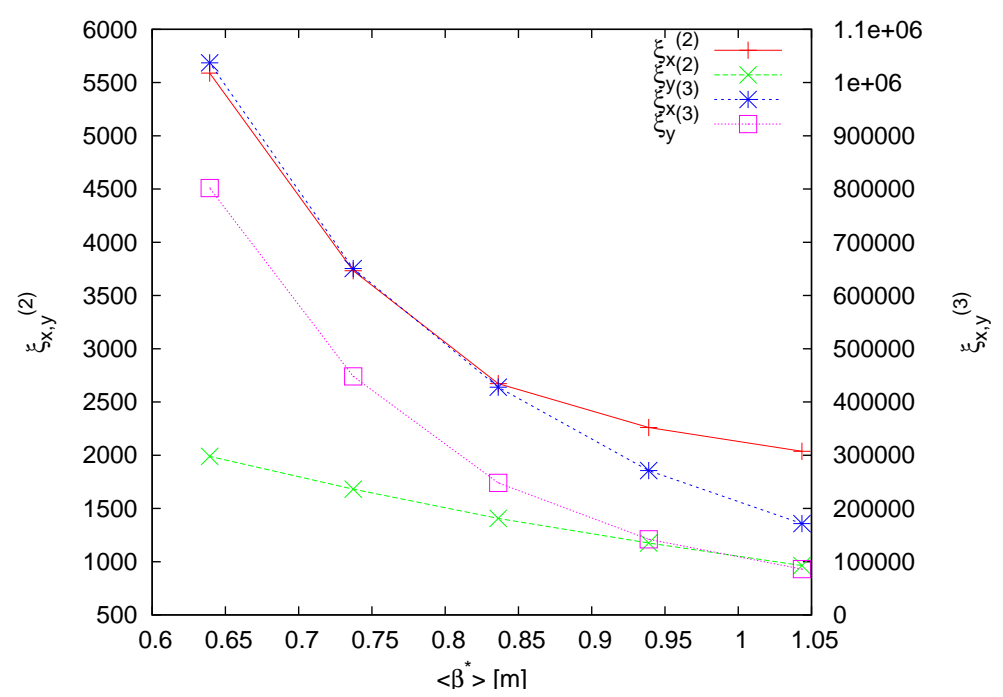

Figure 11: Chromaticities versus $\beta^{*}$ for $2010100 \mathrm{GeV} \mathrm{Au}-\mathrm{Au}$ run Yellow ring lattices

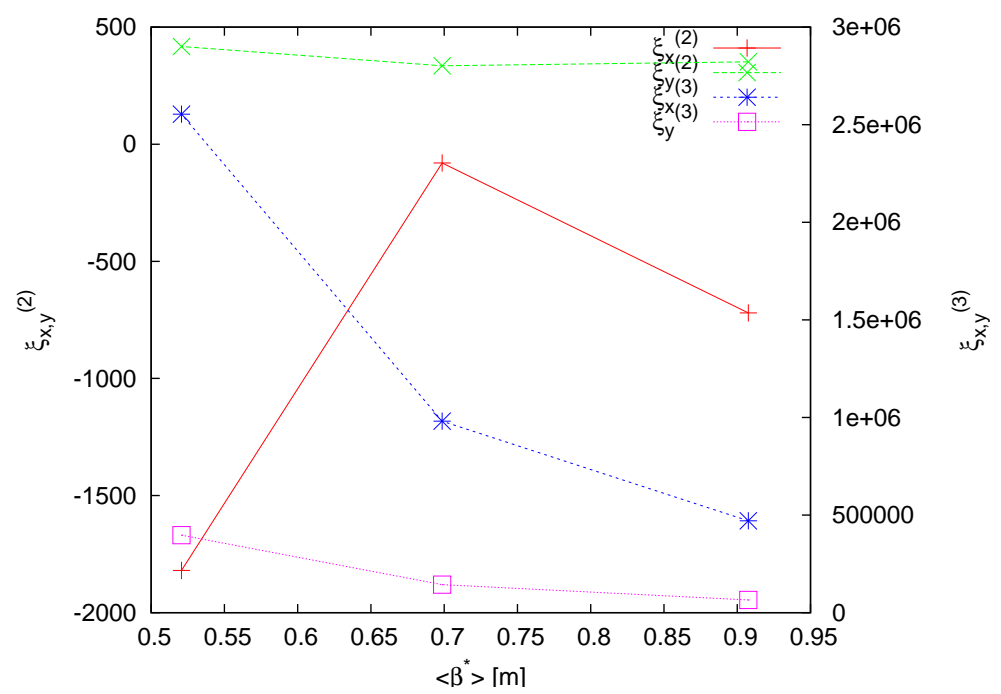

Figure 12: Chromaticities versus $\beta^{*}$ for $2009100 \mathrm{GeV}$ p-p run Blue ring lattices with $\xi^{(2)}$ corrected 


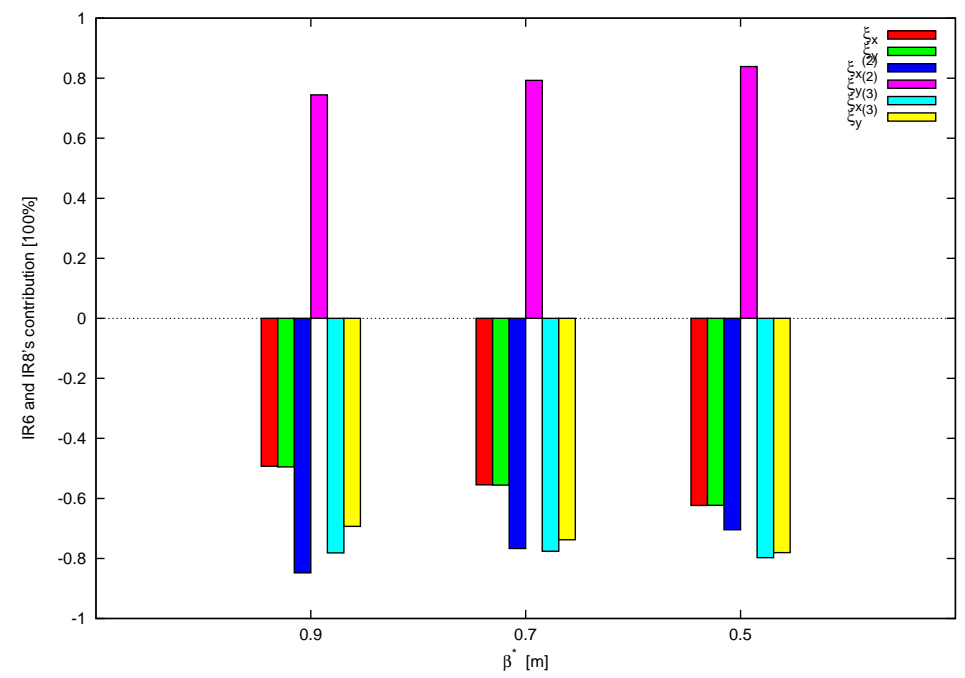

Figure 13: IR6 and IR8's contributions to chromaticities versus $\beta^{*}$ at IP6 and IP8 for the $2009100 \mathrm{GeV}$ p-p run Blue ring lattices

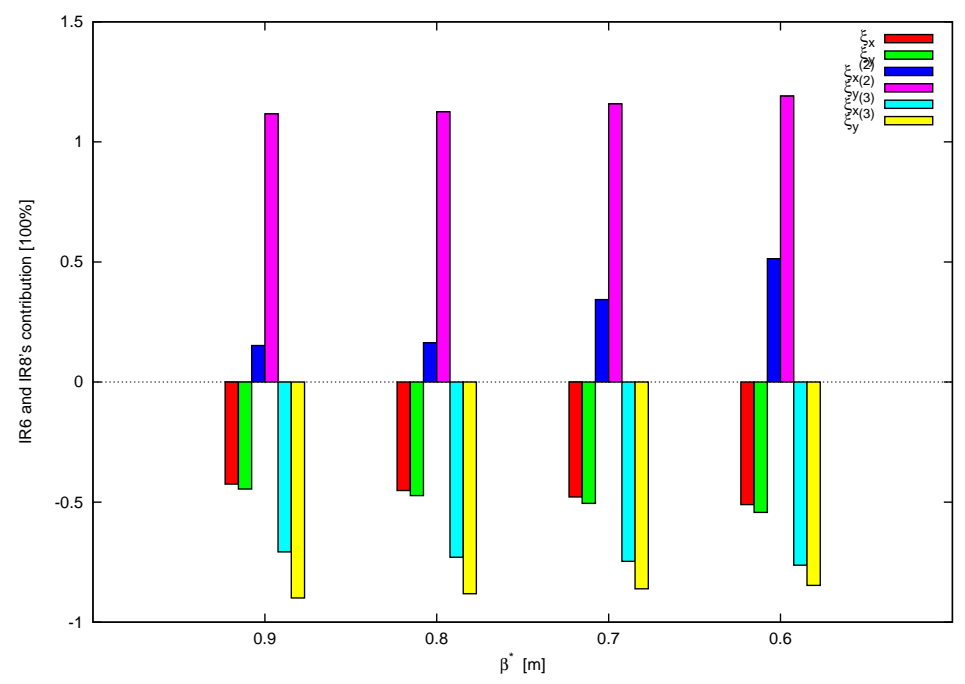

Figure 14: IR6 and IR8's contributions to linear and nonlinear chromaticities versus $\beta^{*}$ at IP6 and IP8 for the $2010100 \mathrm{GeV} \mathrm{Au}-\mathrm{Au}$ run Yellow ring lattices 